\title{
Transferencia de recursos alimentarios entre diferentes ambientes del ecosistema marino
}

\author{
Transfer of food resources among different environments in the marine ecosystem
}

\author{
SEBASTIAN R. RODRIGUEZ
}

Departamento de Ecología, P. Universidad Católica de Chile, Casilla 114-D, Santiago, Chile, e-mail: esrodrig@genes.bio.puc.cl

\begin{abstract}
RESUMEN
Por décadas los ecólogos han centrado sus estudios en interacciones que involucran elementos de un mismo sistema, poniendo poco énfasis en aquellas que involucran elementos de ambientes aledaños. Estudios desarrollados en los últimos años han vuelto a llamar la atención respecto de la frecuencia con la que ocurre transferencia de energía (en la forma de nutrientes o alimento) entre sistemas, ambientes y/o hábitats ecológicos, y las consecuencias de estos aportes energéticos a nivel poblacional o comunitario a distintas escalas espaciales y temporales en los sistemas involucrados. En la presente revisión se describen las vías de transferencia de energía más comunes de observar en el ecosistema marino, poniendo especial énfasis en el flujo de recursos tróficos (i.e., algas a la deriva y detritus) desde bosques de macroalgas pardas submareales hacia ambientes intermareales en los sistemas templados.
\end{abstract}

Palabras clave: flujo de recursos tróficos, interacción submareal-intermareal, macroalgas a la deriva, detritus, sistemas templados.

\begin{abstract}
For decades ecologists have focused their studies in interactions among elements of the same system, putting low emphasis in those that involve elements of border environments. Studies carried out in the last years have called the attention respect the frequent ocurrence of energy transfer (as nutrients or food) among ecological systems, environments and/or habitats, and the consequences of these energy contributions at population or community levels and at different spatial and temporal scales. In this review the ways of energy transfer more commonly observed in the marine ecosystem are described. The flow of trophic resources (i.e., drift algae and detritus) from subtidal kelps to intertidal environments in template systems, are emphasized.
\end{abstract}

Key words: flow of trophic resources, subtidal-intertidal interaction, drift kelp, detritus, template systems.

\section{INTRODUCCION}

El problema de escala es un tema que resulta fundamental en ciencia, aunque sólo reciéntemente ha recibido un reconocimiento explícito en ecología (Wiens 1989). Por muchos años los ecólogos han atribuido los patrones observados en sus sistemas de estudio a la acción de procesos que actúan exclusivamente a escala local. En su mayoría, los estudios clásicos de competencia (Schoener 1983, Connell 1983), depredación (Paine 1980, Schoener 1989), o parasitismo (Price et al. 1986) dan cuenta de interacciones entre organismos de un mismo hábitat, y ponen poco énfasis en la influencia de factores y procesos que provienen de hábitats vecinos. Como consecuencia de esta tendencia, los diferentes componentes del ecosistema marino (e.g., arrecifes rocosos submareales e intermareales, bosques de macroalgas pardas, estuarios, etc.) han sido generalmente analizados como unidades ecológicas discretas y distintas entre sí (Fairweather \& Quinn 1992). Sin embargo, en la última década ha comenzado a ser reconocido que diferentes escalas de tiempo y espacio generan diferentes patrones ecológicos (Dayton et al. 1992), que patrones más robustos y predecibles aparecen a medida que se aumenta la escala de análisis (Levin 1992), y que fenómenos emergentes a grandes escalas espaciales pueden contribuir en forma importante a la 
generación de patrones de pequeña escala (Rickleffs 1987, Underwood \& Fairweather 1989, Menge \& Olson 1990). En otras palabras, se ha tomado conciencia de que los patrones observados en muchas comunidades biológicas dependen de una interacción compleja entre procesos espaciales de gran escala y procesos locales.

La necesidad de ampliar el horizonte de observación para lograr un entendimiento más acabado de los patrones observados a escala local, comenzó a tener un mayor impulso a fines de los años ochenta, principalmente por el creciente número de trabajos en el área de la ecología del abastecimiento (i.e., "supply-side-ecology") y el reconocimiento de que los sistemas ecológicos son en muchos casos sistemas abiertos (Lewin 1986, Young 1987, Underwood \& Fairweather 1989). Estudios en esta línea permitieron crear conciencia de que en algunos sistemas marinos costeros los factores físicos y/o biológicos que afectan a los estados larvales en la columna de agua podían ser tanto o más importantes que las interacciones biológicas que afectan a los adultos a escala local en la determinación de los patrones de una comunidad (veáse Roughgarden et al. 1994). Sin embargo, y trascurridos los años, la gran mayoría de los estudios han centrado su atención sólo en esta problemática no poniendo énfasis suficiente en otras instancias de interacción entre sistemas o hábitats vecinos igualmente importantes, como los aportes energéticos en la forma de nutrientes o alimento.

APORTES ENERGETICOS ENTRE SISTEMAS, AMBIENTES Y HABITATS ECOLOGICOS VECINOS

El paisaje marino puede definirse como un mosaico de parches de hábitat espacialmente discretos y reconocibles, definidos a una variedad de escalas jerárquicas (Jones \& Andrew 1992). En términos globales, la ecología del paisaje marino enfatiza entre otros aspectos la persistencia de parches, la naturaleza de los bordes y especialmente la conexión entre parches a diferentes escalas. La necesidad de considerar la influencia de factores externos al sistema o hábitat en estudio se ha visto reciéntemente reforzada por el reconocimiento más explícito de que la transferencia de energía de un lugar a otro es un fenómeno que ocurre con gran frecuencia en la naturaleza (e.g., Polis et al. 1997). Es en esta perspectiva que algunos ecólogos han vuelto a llamar la atención respecto de lo importante que puede llegar a ser el flujo de nutrientes orgánicos, detritus o alimento entre distintos sistemas o entre distintos hábitats de un sistema para la determinación de patrones ecológicos en una comunidad local (e.g., Bustamante et al. 1995, Polis et al. 1995). A continuación se hace mención a algunas de las instancias más importantes de contribución energética entre diferentes sistemas, ambientes y/o hábitats ecológicos vecinos (véase Tabla 1 ).

\section{Sistemas terrestre y marino}

Los ecosistemas terrestre y marino son dos ambientes en los que con frecuencia es posible observar un importante flujo de energía. Por ejemplo, nutrientes de sedimentos erosionados, energía en la forma de materia orgánica en descomposición, y materia en la forma de sedimentos son frecuentemente transportados hacia el mar a través de los ríos (Fairweather \& Quinn 1992). Asimismo, gran parte de la producción de hábitats estuarinos de fondos blandos con vegetación y el detritus de ríos es exportado hacia zonas oceánicas (i.e., "outwelling hypothesis", véase Dame et al. 1986, citado en Fairweather \& Quinn 1992). En ausencia de surgencias, esta exportación sería una importante fuente de nutrientes y energía para los ensambles pelágicos y bentónicos costeros (véase Jones \& Andrew 1992). Risk et al. (1994), observaron que dos especies de corales cercanos a la costa obtenían cerca de un tercio de sus nutrientes de fuentes terrestres (i.e., materia orgánica disuelta y particulada), y que esta influencia disminuía paulatinamente a mayores profundidades en La Gran Barrera de Arrecife en Australia. Es importante hacer notar que la interacción entre los sistemas marinos y terrestres a través de los ríos también puede tener connotaciones negativas en algunos casos. Períodos de turbiedad del agua de mar como resultado de descargas terrestres han sido implicados en la mortalidad masiva de macroalgas en aguas profundas donde la penetración de luz es baja (Jones \& Andrew 1992). Asimismo, la descarga en el mar de agua dulce durante períodos de fuertes lluvias ha sido sugerida como un factor de mortalidad de erizos en arrecifes cercanos a estuarios (Andrew \& Underwood 1989).

Otra ruta de transferencia energética entre los sistemas terrestre y marino, en uno u otro sentido, se da a través de las aves (Polis et al. 1997). Heatwole (1971), fue uno de los primeros en describir la importancia de estos vertebrados en la transferencia de energía desde el mar a comunidades insulares del Mar Coral. Las aves marinas pueden actuar como vectores de energía y nutrientes en forma directa a través del guano (Wootton 1991, Heatwole 1971), o de manera indirecta a través de sus parásitos y sus carcasas, 
las cuales son consumidas por artropodívoros y carroñeros terrestres respectivamente (Polis \& Hurd 1995 a, b). Por último, las algas y restos de invertebrados y vertebrados marinos a la deriva depositados en la costa también representan una fuente de energía de origen marino que puede ser utilizado por organismos terrestres. Anderson \& Polis (1998), a través de análisis de isótopos estables, demostraron que dichos restos son consumidos por carroñeros y detritívoros los cuales a su vez son depredados por arañas y escorpiones. A través de esta vía el material marino se incorporaría a la trama trófica terrestre.

\section{Ambientes marinos adjacentes}

Dentro de los ambientes marinos, los sistemas de corriente global y el movimiento de aguas frías ricas en nutrientes hacia la superficie a través de las surgencias son instancias que permiten un flujo importante de energía entre mar abierto y los ambientes costeros (Bernal \& Ahumada 1985). Este aumento en los niveles de nutrientes en muchas ocasiones está ligado a importantes aumentos en la productividad. También materia y energía son a menudo transferidas entre la columna de agua (especialmente la zona fótica) y el bentos marino. Por ejemplo, muchos organismos (e.g., peces) pueden transportar materia orgánica desde el plancton hacia hábitats bentónicos, vía ingestión en la primera y defecación en la última (Bray et al. 1981, Rothans \& Miller 1991). Bray et al. (1981) observaron que una especie de pez que consume zooplancton en la columna de agua durante el día y busca protección en arrecifes rocosos durante la noche en las costas del sur de California, era capaz de exportar hacia estos am-

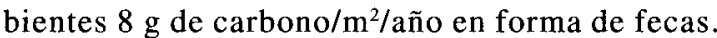
Este transporte de carbono orgánico sería predecible en el tiempo y en el espacio dado la tendencia de los individuos a retornar a las mismas grietas durante el período inactivo. Asimismo, este material se incorporaría a la trama trófica de la comunidad al ser consumido por especies detritívoras (principalmente camarones) que forman parte de la dieta de algunos peces (Rothans \& Miller 1991). Por último, una transferencia de materia y energía entre estos ambientes puede darse a través de la llamada "nieve marina" (e.g., agregados de células de plantas, Alldredge \& Silver 1988) o incluso a través de agregados de algas a la deriva, que al perder sus estructuras flotantes y hundirse (Harrold \& Lisin 1989), son capaces de transferir carbono al bentos (Kingsford 1995). El efecto de agregados de macroalgas a la deriva, en especial en zonas de convergencia y retención (determinadas por rasgos oceanográficos), sobre los ensambles bentónicos inmediatamente por debajo de estas zonas ha sido por

TABLA 1

Transferencia de energía en la forma de nutrientes o alimento entre diversos sistemas, ambientes y/o hábitats ecológicos

Transfer of energy as nutrient or food among different ecological systems, environments and/or habitats

\begin{tabular}{ll} 
Sistemas, Ambientes o Hábitats & Vías de transferencia \\
\hline Sistemas Terrestre y Marino & - Flujos de nutrientes, materia orgánica en descomposición y sedimentos desde \\
& la tierra al mar a través de rios, y utilización por organismos marinos costeros. \\
- & Depósito de guano, parasitos o restos sin vida de aves marinas en la costa, y \\
& utilización por artropodívoros y carroñeros terrestres. \\
- & Arribo de algas o restos de invertebrados y vertebrados marinos a la deriva en \\
& la costa, y utilización por carroñeros y detritivoros terrestres.
\end{tabular}

Ambientes Oceánico y Costero - Flujo de nutrientes a través de surgencias.

Ambientes Pelágico y Bentónico - Flujo de materia y energía entre columna de agua y fondo marino a través de "nieve marina" o restos de algas y animales sin vida.

- Defecación en el bentos de organismos de alimentación pelágica.

Habitats vecinos en arrecifes rocosos - Transporte de algas a la deriva desde arrecifes rocosos a sedimentos blandos y utilización de este recurso por especies de este habitat.

- Flujo de algas a la deriva o detritus generado a partir de macroalgas pardas de arrecifes rocosos hacia habitats vecinos a los de producción, y utilización por invertebrados o especies filtradoras. 
muchos años ignorado, y representa un área que sin duda tendrá que ser abordada en el futuro cercano (Kingsford 1995).

\section{Hábitats submareales e intermareales}

Dado que los hábitats submareales e intermareales coexisten a pocos metros en el ecosistema marino, es esperable que entre ellos también exista una importante y permanente transferencia de energía. En el caso particular de las costas templadas donde los bosques de macroalgas pardas submareales son hábitats bastante comunes (Dayton 1985), una parte importante de la materia orgánica producida en estos ambientes, ya sea como algas a la deriva o detritus, puede ser transferida a los ambientes intermareales e incorporarse a través de distintas vías en sus flujos de energía (Fig. 1).

\section{TRANSFERENCIA DE RECURSOS TROFICOS DESDE BOSQUES DE MACROALGAS PARDAS HACIA HABITATS INTERMAREALES}

\section{Macroalgas a la deriva}

Generación y transporte hacia sistemas intermareales: Una serie de factores abióticos y bióticos pueden estar involucrados en la producción de algas a la deriva a través del desprendimiento de macroalgas de bosques submareales. Dentro de los factores abióticos se pueden mencionar la fuerza del movimiento del agua y el desprendimiento por enredo con materiales a la deriva (Rosenthal et al. 1974, Dayton \& Tegner 1984, Dayton et al. 1984, 1992, Schiel \& Foster 1986). Por su parte, dentro de los factores bióticos cabe destacar el debilitamiento de discos adhesivos o estipes de macroalgas por ramoneo de pastoreadores (e.g., erizos) (Koehl \& Wainwright 1977, Dayton et al. 1984, 1992).

Algunos autores han descrito que la producción de plantas a la deriva en praderas de macroalgas submareales y su posterior transporte hacia zonas costeras varía temporalmente. Mientras un alto porcentaje de macroalgas desprendidas se acumulan en verano al interior de bosques submareales como consecuencia del menor movimiento del agua, una gran cantidad de algas a la deriva es exportada fuera del bosque durante el invierno por efecto de las corrientes (Harrold \& Reed 1985, Tegner \& Dayton 1987, 1991, Dayton et al. 1992). Este patrón fue originalmente descrito por Gerard (1976), quien al cuantificar las algas a la deriva depositadas en parcelas permanentes de 10

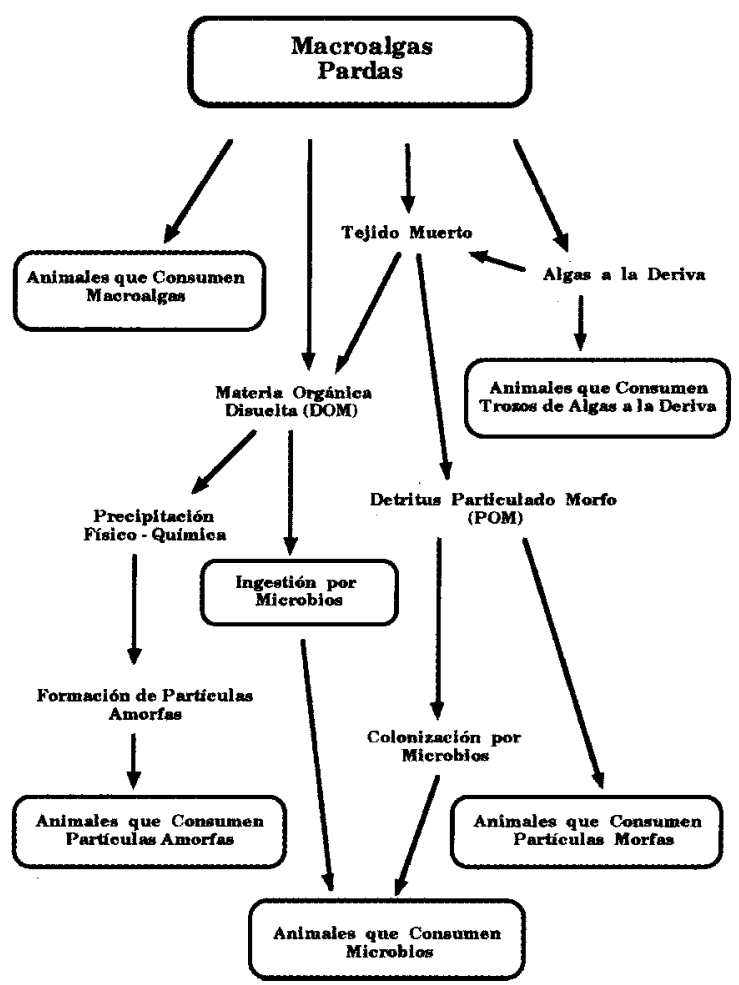

Fig. 1. Utilización de macroalgas pardas por organismos marinos.

Use of brown macroalgae by marine organisms.

$\mathrm{m}^{2}$ al interior de una pradera de macroalgas pardas en Punta Cabrillo, California, observó una mayor biomasa durante el verano y principios del otoño. Las algas o frondas que se desprenden en bosques submareales someros pueden ser transportadas a grandes distancias (e.g., 10 a $12 \mathrm{~km} /$ día) y ser depositadas en lugares distantes de aquellos en los cuales se han originado (véase Kingsford 1995), o por el contrario, depositarse en el mismo sistema (como fue observado por Harrold \& Lisin 1989). El viento, las corrientes costeras y las olas internas serían algunos de los mecanismos de transporte más importantes (Kingsford 1995, Harrold \& Lisin 1989).

Utilización por organismos intermareales: Las algas transportadas a la deriva constituyen el alimento primario para numerosas especies de invertebrados marinos como poliquetos (e.g., Magnum et al. 1968, Magnum \& Cox 1971), patelas (e.g., Bustamante et al. 1995), abalones (e.g., Shepherd 1973, Tutschulte \& Connell 1988), erizos (e.g., Castilla \& Moreno 1981, Dayton et al. 1984, Harrold \& Reed 1985) y estrellas (e.g., Gerard 1976). Kim (1992), demostró que algas a la deriva constituyen el item alimenticio primario 
para un poliqueto tubícola que habita en sedimentos blandos adjacentes a un gran bosque de macroalgas pardas en las costas de California. Asimismo, mostró que el tamaño de estos organismos (no así la densidad) se veía afectado en forma importante por la disponibilidad de estas algas. Dado que este poliqueto proporciona la estructura física estabilizadora en estos sedimentos, Kim (1992) concluyó que cambios en la estructura de tamaño de la población de este poliqueto, como resultado de una mayor o menor disponibilidad de algas a la deriva, podía tener consecuencias importantes para toda la comunidad. Por ejemplo, la presencia de organismos de mayor talla podría reducir el movimiento de arena, atraer más y diferentes depredadores o restringir la presencia de organismos infaúnicos de gran tamaño (Kim 1992).

A pesar de que ha sido largamente reconocido que las algas a la deriva pueden representar una fuente de alimento para organismos herbívoros del intermareal (Hawkins \& Hartnoll 1983), muy pocos estudios han intentado cuantificar la importancia de este recurso para organismos de este ambiente y sus consecuencias. En el caso de las playas de arena, las macroalgas son rápidamente colonizadas por anfípodos e insectos, los cuales utilizan el recurso como refugio y/o alimento (e.g., Duarte 1974, Griffiths \& Stenton-Dozey 1981, Behbehani \& Croker 1982, Lavoie 1985, Inglis 1989). Esto es así especialmente para aquellas macroalgas que alcanzan los bordes superiores de las playas y que no pueden ser resuspendidas durante la siguiente marea alta, permaneciendo en esta zona por períodos de días o semanas (Yaninek 1980). Dada la falta de producción primaria in situ en estos ambientes, estas macroalgas representan un aporte orgánico fundamental para muchos consumidores (e.g., anfípodos, véase Duarte 1974, Inglis 1989). Más aún, luego de su descomposición por microbios y procesos de fragmentación abiótica, parte de la energía contenida en estas macroalgas se incorporaría a las cadenas tróficas intersticiales de estos ambientes (Inglis 1989). Por su parte, Bustamante et al. (1995) trabajando con dos especies de patelas ocupadoras dominantes del espacio en el intermareal rocoso de las costas de Sudáfrica, observaron que la exclusión de algas a la deriva provenientes del submareal disminuía la sobrevivencia de una de ellas. Asimismo, demostraron que la densidad de patelas observada no podía ser mantenida por la producción algal epilítica intermareal. De esta manera, las altas densidades de patelas requerían de un recurso alimentario exógeno, el cual estaba dado por el aporte de algas a la deriva de macroalgas submareales. Bustamante et al. (1995) propusieron que dicho "subsidio trófico" tendría profundas repercusiones en la estructura y funcionamiento de la comunidad intermareal, al disminuir la abundancia y diversidad de macroalgas, aumentar la cobertura de coralinas encrustantes, y disminuir la ocupación de espacio por filtradores. Finalmente, Rodríguez (1999) dió cuenta que las macroalgas pardas a la deriva provenientes del intermareal bajo o submareal somero representaban el principal recurso alimentario de erizos de pozas intermareales en las costas de Chile central, y que su nivel de disponibilidad podía tener importantes consecuencias poblacionales al afectar significativamente el desarrollo de tejido gonadal de los individuos. De la misma forma, Rodríguez (1999) propuso que el consumo de algas pardas a la deriva sería capaz de aumentar la abundancia y riqueza taxonómica de macroalgas y disminuir la cobertura de crustosas calcáreas al interior de las pozas intermareales, gatillando con ello patrones de estructura comunitaria similares a aquellos determinados por la ausencia de reclutamiento de erizos juveniles o la presencia de altas tasas de mortalidad de erizos adultos (veáse Watanabe \& Harrold 1991 y Jones et al. 1985 respectivamente).

\section{Detritus}

Generación y utilización por organismos intermareales: Las algas liberan una gran cantidad de materia orgánica disuelta (DOM). Por ejemplo, ha sido calculado que el flujo total de DOM de algunas algas durante el crecimiento y después de la muerte podría aproximarse a un $70 \%$ de su producción neta (Khailov \& Burlakova 1969). Por el contrario, la cantidad de materia orgánica particulada (POM) liberada de macroalgas, así como los agentes involucrados en su generación han sido poco estudiados. Además de la descomposición química y el desgaste físico natural de los tejidos vivos o muertos de las macroalgas, es posible que los organismos herbívoros (e.g., peces) a través de sus fecas también jueguen un papel importante en la liberación de POM a la columna de agua (veáse Rodríguez 1999).

Aún cuando el detritus derivado de macroalgas pardas puede llegar a ser una importante fuente de alimento para numerosos organismos filtradores intermareales (Duggins et al. 1989, Bustamante \& Branch 1995, Rodríguez 1999), escasos son los estudios que han intentado medir el efecto de este recurso en las dinámicas tróficas de este ambiente. Rodríguez (1999) observó que dos de las especies filtradoras más conspicuas del intermareal rocoso de la costa de Chile central presentaban 
valores de isótopos de carbono más cercanos a aquellos de particulas de materia orgánica derivada de macroalgas pardas de bosques submareales que al del fitoplancton. Bustamante \& Branch (1995), al estudiar las relaciones tróficas existentes entre el sistema intermareal y submareal de una comunidad en las costas de Sudáfrica, encontraron que el detritus producido por macroalgas submareales representaba el $65 \%$ del POM presente en la columna de agua. Luego de medir la utilización de este detritus en especies filtradoras intermareales, dichos autores concluyeron que éste representaba la principal fuente de carbono y nitrógeno orgánico para estos organismos. Por su parte, Duggins et al. (1989) demostraron que las tasas de crecimiento de filtradores bentónicos tanto intermareales como submareales eran mayores en presencia que en ausencia de detritus orgánico de macroalgas submareales en islas del archipiélago Aleutiano (Alaska). A través de un análisis de isótopos de carbono estable los autores pudieron confirmar que el carbono proveniente de las macroalgas submareales se encontraba a lo largo de las tramas tróficas costeras (Duggins et al. 1989).

Es importante señalar que la disponibilidad de detritus para un consumidor depende en gran medida de la fuente de la cual proviene el detritus y de la edad que este detritus posee (Tenore 1977 a, b). Tenore \& Hanson (1980), midiendo la disponibilidad de detritus de diferentes fuentes y edades para una especie de poliqueto en experimentos de laboratorio, observaron que la descomposición del particulado (dada principalmente por la biomasa de microbios, la cual aumenta con la edad) era más rápida para algunas microalgas y macroalgas que para una especie de planta vascular. Asimismo, observaron que mientras más rápida era la descomposición del detritus mayor era la cantidad de éste utilizada en el crecimiento del poliqueto. En base a estos resultados, los autores concluyeron que era esperable una mayor producción de macroconsumidores en sistemas sostenidos por detritus producido por macroalgas marinas que en aquellos que reciben un aporte comparable de detritus proveniente de plantas vasculares. Este hecho explicaría la baja producción de macroconsumidores asociados a pantanos salobres ("salt-marsh") (Tenore \& Hanson 1980).

Dado que la edad del detritus juega un papel importante en su eventual utilización por organismos marinos, es importante considerar la escala cuando se intenta determinar el rol del detritus proveniente de algas en la energética de los sistemas costeros. Por ejemplo, Duggins \& Eckman (1994), no detectaron un papel importante del detritus proveniente de praderas de Agarum submareales en el crecimiento de filtradores bentónicos que habitan bajo el dosel de estos bosques en el archipiélago de San Juan en las costas del estado de Washington. Este hecho habría estado dado por la presencia en Agarum de compuestos fenólicos que afectarían la asimilación por parte de los filtradores. Como los autores observaron que el contenido fenólico de partículas derivadas de esta alga disminuía rápidamente con la edad, era muy probable que su contribución como partícula alimentaria para el crecimiento de filtradores sólo se diera a escalas regionales y no locales (i.e., dentro de la pradera) (Duggins \& Eckman 1994).

La utilización diferencial de carbono de diferentes fuentes puede ocurrir de acuerdo a la localización del organismo respecto de esas fuentes (Peterson et al. 1985). Sin embargo, los organismos que utilizan el detritus proveniente de algas no siempre lo hacen de la fuente más cercana disponible. Stephenson et al. (1986) observaron que a excepción de una especie ninguna otra derivaba una cantidad significativa de carbono de las macroalgas del bosque donde habitaban, siendo éste obtenido posiblemente del fitoplancton. Por último, la utilización de carbono de una u otra fuente puede variar de acuerdo a la disponibilidad de éstas en diferentes épocas del año. Dunton \& Schell (1987) observaron cambios estacionales en la utilización de carbono de diferentes fuentes por parte de organismos de un bosque de macroalgas en Alaska (i.e., aumento de la dependencia de carbono proveniente de macroalgas durante el invierno cuando el fitoplancton es poco abundante o está ausente).

\section{CONCLUSIONES}

En la presente revisión se pone en evidencia que la gran mayoría de los sistemas, ambientes y/o hábitats marinos se encuentran conectados a través de la transferencia de energía en la forma de nutrientes o recursos tróficos. Sin embargo, en el caso particular de las costas templadas, el aporte energético existente entre hábitats vecinos es bastante desconocido. Sólo en los últimos años, unos pocos trabajos han llamado la atención respecto de la importancia de las algas a la deriva y en menor medida del material orgánico particulado derivado de bosques de macroalgas pardas submareales como fuente alimentaria de organismos invertebrados intermareales. A pesar de lo anterior, la biomasa de algas depositadas en los sistemas intermareales, así como los patrones espaciales y temporales de arribo son prácticamente desconocidos. De la misma forma, y a pesar de que el consumo de algas a 
la deriva por invertebrados es un fenómeno bastante común en los ambientes marinos, su utilización por organismos intermareales ha recibido poca atención. Más aún, en escasas ocasiones ha sido medida la magnitud de este consumo o el grado de dependencia de estos organismos para con esta fuente de alimento. Finalmente, existe una evidente falta de información respecto de las consecuencias que estos subsidios tróficos pueden llegar a tener a distintos niveles de organización biológica, en especial en la estructuración de las comunidades receptoras. Estudios en los últimos años han demostrado que la dinámica de tramas tróficas de algunos sistemas pueden depender de recursos alimentarios provenientes de sistemas vecinos (Polis et al. 1995, 1997). Por ejemplo, recursos tróficos marinos pueden llegar a ser claves para mantener altos niveles de producción en algunos sistemas intermareales (e.g., alta biomasa de patelas; Bustamante et al. 1995) o incluso en ambientes terrestres (e.g., alta densidad de arañas; ver Anderson \& Polis 1998, Polis \& Hurd 1995 b).

Hoy más que nunca parece fundamental en ecología el aumentar la escala de observación cuando se trata de entender procesos involucrados en la determinación de patrones a escala local. Sólo una visión de los sistemas, ambientes y/o hábitats marinos como entidades interactivas y en permanente contribución energética permitirá entender de mejor manera los patrones observados en nuestros sistemas de estudio y de esta forma el complejo comportamiento de los sistemas ecológicos.

\section{AGRADECIMIENTOS}

El presente trabajo, que representa el marco conceptual de la Tesis Doctoral del autor, fue parcialmente financiado por el Proyecto FONDECYT 2960015. Durante la elaboración de este manuscrito S.R.R. fue becario de Fundación Andes. El autor agradece a J.M. Fariña, al Dr. F.P. Ojeda, al Dr. Carlos Moreno y a dos revisores anónimos cuyas sugerencias y comentarios ayudaron a mejorar sustancialmente el manuscrito final.

\section{LITERATURA CITADA}

ALLDREDGE AL \& MW SILVER (1988) Characteristics, dynamics and significance of marine snow. Progress in Oceanography 20: 41-82.

ANDERSON WB \& GA POLIS (1998) Marine subsidies of island communities in the Gulf of California: evidence from stable carbon and nitrogen isotopes. Oikos 81: 75-80.
ANDREW NL \& AJ UNDERWOOD (1989) Patterns of abundance of the sea urchin Centrostephanus rodgersii (Agassiz) on the central coast of New South Wales, Australia. Journal of Experimental Marine Biology and Ecology 131: 61-80.

BEHBEHANI MJ \& RA CROKER (1982) Ecology of beach wrack in northern New England with special reference to Orchestia platensis. Estuarine Coastal Shelf Science 15: 611-620.

BERNAL P \& R AHUMADA (1985) Ambiente oceánico. En: Soler F (ed) Medio ambiente en Chile: 55-105. Ediciones Universidad Católica, Santiago.

BRAY RN, AC MILLER \& GG GEESEY (1981) The fish connection: a trophic link between planktonic and rocky reef communities? Science 214: 204-205.

BUSTAMANTE RH \& GM BRANCH (1995) The dependence of intertidal consumers on kelp-derived organic matter on the west coast of South Africa. Journal of Experimental Marine Biology and Ecology 196: 1 - 28.

BUSTAMANTE RH, GM BRANCH \& S AEKHOUT (1995) Maintenance of an exceptional grazer biomass on South African intertidal shores: trophic subsidy by subtidal kelps. Ecology 76: 2314-2329.

CASTILLA JC \& CA MORENO (1981) Sea urchins and macroalgae: an experimental test of their ecological relations in southern Chile. En: Lawrence JM (ed) Echinoderms: 257-263. Proceedings of the International Echinoderm Conference, Tampa Bay, USA.

CONNELL JH (1983) On the prevalence and relative importance of interspecific competition: evidence from field experiments. American Naturalist 122: 661-696.

DAME R, T CHRZANOWSKI, K BILDSTEIN, B KJERVE, H MCKELLER, D NELSON, J SPURRIER, S STANCYK, H STEVENSON, J VERNBERG \& $R$ ZINGMARK (1986). The outwelling hypothesis and North Inlet, South Carolina. Marine Ecology Progress Series 33: 217-229.

DAYTON PK (1985) Ecology of kelp communities. Annual Review of Ecology and Systematic 16: 215-245.

DA YTON PK \& MJ TEGNER (1984) Catastrophic storms, E1 Niño and patch stability in a southern California kelp community. Science 224: 283 -285.

DAYTON PK, V CURRIE, T GERRODETTE \& BD KELLER (1984) Patch dynamics and stability of some California kelp communities. Ecological Monographs 54: 253-289.

DAYTON PK, MJ TEGNER, PE PARNELL \& PB EDWARDS (1992) Temporal and spatial patterns of disturbance and recovery in a kelp forest community. Ecological Monographs 62: 421-445.

DUARTE WE (1974) Orchestoidea tuberculata Nicolet, 1849 como organismo desintegrador de algas (Crustacea, Amphipoda, Talitridae). Noticiero Mensual del Museo Nacional de Historia Natural (Chile) 220-221: 3-9.

DUGGINS DO \& JE ECKMAN (1994) The role of kelp detritus in the growth of benthic suspension feeders in an understory kelp forest. Journal of Experimental Marine Biology and Ecology 176: 53 - 68. 
DUGGINS DO, CA SIMENSTAD \& JA ESTES (1989) Magnification of secondary production by kelp detritus in coastal marine ecosystems. Science 245: 170-173.

DUNTON KH \& DM SCHELL (1987) Dependence of consumers on macroalgal (Laminaria solidungula) carbon in an arctic kelp community: $\delta^{13} \mathrm{C}$ evidence. Marine Biology 93: 615-625.

FAIRWEATHER PG \& GP QUINN (1992) Seascape ecology: the importance of linkages. En: Battershill CN (ed) Proceedings of the Second International Temperate Reef Symposium: 77-83, Auckland, New Zealand.

GERARD VA (1976) Some aspects of material dynamics and energy flow in a kelp forest in Monterrey Bay, California. Ph.D. Thesis, University of California (Santa Cruz). 173 pp.

GRIFFITHS CL \& J STENTON-DOZEY (1981) The fauna and rate of degradation of stranted kelp. Estuarine Coastal Shelf Science 12: 645-653.

HARROLD C \& S LISIN (1989) Radio-tracking rafts of giant kelp: local production and regional transport. Journal of Experimental Marine Biology and Ecology 130: 237-251.

HARROLD C \& DC REED (1985) Food availability, sea urchin grazing and kelp forest community structure. Ecology 66: $1160-1169$.

HAWKINS SJ \& RG HARTNOLL (1983) Grazing of intertidal algae by marine invertebrates. Oceanography and Marine Biology Annual Review 21: 195-282.

HEATWOLE H (1971) Marine-dependent terrestrial biotic communities on some cays in the Coral Sea. Ecology 52: 363-366.

INGLIS G (1989) The colonisation and degradation of stranded Macrocystis pyrifera (L.) C. Ag. by the macrofauna of a New Zealand sandy beach. Journal of Experimental Marine Biology and Ecology 125: 203217.

JONES GM, AJ HEBDA, RE SCHEIBLING \& RE MILLER (1985) Histopathology of the disease causing mass mortality of sea urchins (Strongylocentrotus droebachiensis) in Nova Scotia. Journal of Invertebrate Pathology 45: 260-271.

JONES GP \& NL ANDREW (1992) Temperate reefs and the scope of seascape ecology. En: Battershill CN, (ed) Proceedings of the Second International Temperate Reef Symposium: 63-76 Auckland, New Zealand.

KHAILOV KM \& ZP BURLAKOVA (1969) Release of dissolved organic matter by marine seaweeds and distribution of their total organic production to inshore communities. Limnology and Oceanography 14:521527.

KIM SL (1992) The role of drift kelp in the population ecology of a Diopatra ornata Moore (Polychaeta: Onuphidae) ecotone. Journal of Experimental Marine Biology and Ecology 156: 253-272.

KINGSFORD MJ (1995) Drift algae: a contribution to near-shore habitat complexity in the pelagic environment and an attractant for fish. Marine Ecology Progress Series 116: 297-301.

KOEHL MAR \& SA WAINWRIGHT (1977) Mechanical adaptations of a giant kelp. Limnology and Oceanography 22: $1067-1071$.
LAVOIE DR (1985) Population dynamics and ecology of beach wrack macroinvertebrates of the central California coast. Bulletin S. California Academy of Science 84: 1-22.

LEVIN SA (1992) The problem of pattern and scale in ecology. Ecology 73: 1943-1967.

LEWIN R (1986) Supply-side ecology. Science 234: 2527.

MAGNUM CP \& CD Cox (1971) Analysis of the feeding response in the onuphid polychaete Diopatra cuprea . Biological Bulletin 145: 215-229.

MAGNUM CP, SL SANTOS \& WR RHODES Jr (1968) Distribution and feeding in the onuphid polychaete Diopatra cuprea. Marine Biology 21: 33-40.

MENGE BA \& AM OLSON (1990) Role of scale and environmental factors in regulation of community structure. Trends in Ecology and Evolution 5: 52-57.

PAINE RT (1980) Food webs: linkage, interaction strength and community infrastructure. Journal of Animal Ecology 49: 667-685.

PETERSON BJ, RW HOWARTH \& RH GARRIT (1985) Multiple stable isotopes used to trace the flow of organic matter in estuarine food webs. Science 227 : $1361-1363$

POLIS GA \& SD HURD (1995 a) Allochthonous input across habitats, subsidized consumers, and apparent trophic cascades: examples from the ocean-land interface. En: Polis GA \& K Winemiller (eds) Food webs: integration of patterns and dynamics: $275-285$. Chapman \& Hall, London.

POLIS GA \& SD HURD (1995 b) Extraordinarily high spider densities on islands: flow of energy from the marine to terrestrial food webs and the absence of predation. Proceedings from the National Academy of Science of the United States 92: 4382-4386.

POLIS GA, RD HOLT, BA MENGE \& KO WINEMILLER (1995) Time, space, and life history: influences on food webs. En : Polis GA \& K Winemiller (eds) Food webs: integration of patterns and dynamics: $435-460$. Chapman \& Hall, London.

POLIS GA, WB ANDERSON \& RD HOLT (1997) Toward an integration of landscape and food web ecology: the dynamics of spatially subsidized food webs. Annual Review of Ecology and Systematic 28: 289-316.

PRICE PW, M WESTOBY, B RICE, PR ATSATT, RS FRITZ, JN THOMPSON \& K MOBLEY (1986) Parasite mediation in ecological interactions. Annual Review of Ecology and Systematic 17: 487-505.

RICKLEFFS RE (1987) Community diversity: relative roles of local and regional processes. Science 235: 161-171.

RISK MJ, PW SAMMARCO \& HP SCHWARCZ (1994) Cross-continental shelf trends in $\delta^{13} \mathrm{C}$ in coral on the Great Barrier Reef. Marine Ecology Progress Series 106: 121-130.

RODRIGUEZ SR (1999) Subsidios tróficos en ambientes marinos: la importancia de las macroalgas pardas a la deriva como fuente exógena de recursos alimentarios para el erizo Tetrapygus niger (Echinodermata: Echinoidea) en el intermareal rocoso de la costa de Chile central. Tesis de Doctorado, Facultad de Ciencias Biológicas, Pontificia Universidad Católica de Chile, Santiago. 172 pp. 
ROSENTHAL RJ, WD CLARKE \& PK DAYTON (1974) Ecology and natural history of a stand of giant kelp, Macrocystis pyrifera, off del Mar, California. Fishery Bulletin 72: 670-684.

ROTHANS TC \& AC MILLER (1991) A link between biologically imported particulate organic nutrients and the detritus food web in reef communities. Marine Biology 110: 145-150.

ROUGHGARDEN J, T PENNINGTON \& S ALEXANDER (1994) Dynamics of the rocky intertidal zone with remarks on generalizations in ecology. Philosophical Transactions of the Royal Society of London B, Biological Sciences 343: 79-85.

SCHIEL DR \& MS FOSTER (1986) The structure of subtidal algal stands in temperate waters. Oceanography and Marine Biology Annual Review 24: 265 307.

SCHOENER TW (1983) Field experiments on interspecific competition. American Naturalist 122: 240-285.

SCHOENER TW (1989) Food webs from the small to the large. Ecology 70: 1559-1589.

SHEPHERD SA (1973) Competition between sea urchins and abalone. Australian Fisheries 32: $4-7$

STEPHENSON RL, FC TAN \& KH MANN (1986) Use of stable carbon isotope ratios to compare plant material and potential consumers in a seagrass bed and a kelp bed in Nova Scotia, Canada. Marine Ecology Progress Series 30: 1-7.

TEGNER MJ \& PK DAYTON (1987) Eł Niño effects on southern California kelp forest communities. Advances in Ecological Research 17: 243-279.

TEGNER MJ \& PK DAYTON (1991) Sea urchins, El Niño, and the long term stability of southern California kelp forest communities. Marine Ecology Progress Series 77: 49-63.
TENORE KR (1977 a) Growth of Capitella capitata cultured on various levels of detritus derived from different sources. Limnology and Oceanography 22 : 937-942.

TENORE KR (1977 b) Utilization of aged detritus derived from different sources by the polychaete Capitella capitata. Marine Biology 44: 51-55.

TENORE KR \& RB HANSON (1980) Availability of detritus of different types and ages to a polychaete macroconsumer, Capitella capitata . Limnology and Oceanography 25: 553-558.

TUTSCHULTE TC \& JH CONNELL (1988) Feeding behavior and algal food of three species of abalone (Haliotis) in southern California. Marine Ecology Progress Series 49: 57-64.

UNDERWOOD AJ \& PG FAIRWEATHER (1989) Supplyside ecology and benthic marine assemblages. Trends in Ecology and Evolution 4: 16-20.

WATANABE JM \& C HARROLD (1991) Destructive grazing by sea urchins Strongylocentrotus spp. in a central California kelp forest: potential roles of recruitment, depth, and predation. Marine Ecology Progress Series 71: 125-141.

WIENS JA (1989) Spatial scaling in ecology. Functional Ecology 3: 385-397.

WOOTTON JT (1991) Direct and indirect effects of nutrients on intertidal community structure: variable consequences of seabird guano. Journal of Experimental Marine Biology and Ecology 151: 139-153.

YANINEK SJ (1980) Beach wrack: phenology of an imported resource and utilization by macroinvertebrates of sandy beaches. Master's Thesis, University of California (Berkeley). $159 \mathrm{pp}$.

YOUNG CM (1987) Novelty of supply-side ecology. Science 235: 415-416. 